\title{
Identificação dos sintomas comportamentais e psicológicos em idosos moradores de uma Instituição de Longa Permanência
}

\section{Identification of Behavioral and Psychological Symptoms in the residents elderly of a Long Stay Institution}

\author{
Mariana Boaro Fernandez Canon ${ }^{1}$, \\ Marcia Maria Pires Camargo Novelli ${ }^{2}$
}

\begin{abstract}
CANON, M. B. F.; NOVELLI, M. M. P. C. Identificação dos sintomas comportamentais e psicológicos em idosos moradores de uma Instituição de Longa Permanência. Rev. Ter. Ocup. Univ. São Paulo, v. 23, n. 1, p. 72-80, jan./abr. 2012.

RESUMO: As doenças crônicas comprometem de forma significativa a qualidade de vida da população idosa e podem ainda, estar associadas a presença de sintomas comportamentais e psicológicos. O objetivo do estudo foi identificar a freqüência e a intensidade dos sintomas comportamentais e psicológicos em idosos institucionalizados, referidos por seus cuidadores formais, relacionando-os com o diagnóstico, a sobrecarga subjetiva e o desgaste proporcionado a esses cuidadores. Foram aplicados três instrumentos de avaliação aos cuidadores formais de uma Instituição de Longa Permanência: o Inventário Neuropsiquiátrico, a Escala de Independência em Atividades da Vida Diária (Escala de Katz) e o Inventário de Sobrecarga de Zarit. Foram avaliados 12 cuidadores formais que responderam por 90 idosos residentes da instituição, 86,7\% dos idosos apresentavam ao menos um sintoma, os mais freqüentes foram: ansiedade (28,9\%), agitação e depressão (ambos com 22,2\%), sendo estes também os mais intensos com 25,6\% (ansiedade), 20\% (agitação) e 17,8\% (depressão). Foram encontradas correlações significativas entre o diagnóstico e a funcionalidade e entre o diagnóstico e os sintomas. O desgaste e a sobrecarga subjetiva correlacionados demonstraram a importância das intervenções nos sintomas comportamentais e psicológicos e a atenção ao cuidador, tanto com relação a informação, quanto ao desgaste e sobrecarga subjetiva que os sintomas acarretam. Destaca-se também que a presença destes contribui para a diminuição da capacidade funcional, que é um marcador de saúde para a população idosa.
\end{abstract}

DESCRITORES: Idoso; Instituição de longa permanência para idosos; Doença crônica; Sintomas comportamentais/psicologia; Cuidadores/psicologia; Questionários/utilização.

\footnotetext{
${ }^{1}$ Terapeuta Ocupacional - Aprimoranda do Programa de Aprimoramento Interdisciplinar em Geriatria e Gerontologia aplicado a Terapia Ocupacional - Hospital do Servidor Público Estadual “Francisco Morato de Oliveira”; E-mail: mcanon.to@gmail.com.

${ }^{2}$ Terapeuta Ocupacional - Professor Adjunto no curso de Terapia Ocupacional da Universidade Federal de São Paulo - Campus Baixada Santista; Líder do Núcleo Interprofissional de Pesquisa e Atendimento no Envelhecimento (NIPAE) Universidade Federal de São Paulo - Campus Baixada Santista. E-mail: mnovelli@uol.com.br
} 


\section{INTRODUÇÃO}

$\mathrm{N}$ os países em desenvolvimento o aumento da população idosa foi muito rápido, contribuindo para o aumento de doenças crônicas e também para as limitações funcionais. Concomitantemente não foram criadas políticas públicas que favorecessem a melhoria na qualidade de vida desta população (NOGUEIRA et al., 2008).

As doenças crônicas comprometem de forma significativa a qualidade de vida da população idosa e estão associadas à senilidade, entre elas destacam-se: a doença de Parkinson, doenças cardiovasculares, hipertensão arterial sistêmica (HAS), diabetes mellitus (DM), doenças pulmonares, entre outras (OMS, 2005).

É importante ressaltar que segundo os dados da Organização Mundial de Saúde (OMS), 60\% da mortalidade mundial em 2005 foi atribuída às doenças crônicas, e se essa tendência for mantida, elas deverão corresponder a $73 \%$ dos óbitos no ano de 2020 (OMS, 2005).

De acordo com o Ministério da Saúde, as doenças crônicas não transmissíveis tornaram-se as principais causas de óbito e incapacidade prematura no Brasil, sendo que 75,5\% dos idosos brasileiros tem alguma doença crônica, segundo a Síntese de Indicadores Sociais de 2009 (IBGE, 2009).

As doenças crônico-degenerativas também são predominantes nesta fase da vida. Dentre elas destacam-se as demências, que segundo o DSM-IV-TR caracteriza-se pelo declínio cognitivo, sendo o da memória o principal, seguido por declínio de outras funções cognitivas, como linguagem, praxia, atenção entre outras, que persistem e interferem de modo significativo no funcionamento ocupacional do indivíduo (APA, 2000).

Os sintomas comportamentais e psicológicos são comuns na demência e também em outros diagnósticos, como seqüela do acidente vascular encefálico, depressão, entre outros. Na demência são classificados como Sintomas Comportamentais e Psicológicos da Demência (SCPD), porém o foco deste estudo foi identificar a presença e intensidade dos sintomas comportamentais e psicológicos em idosos institucionalizados, considerando a demência e outros diagnósticos de doenças crônicas.

Estes sintomas contribuem de forma significativa para a diminuição da qualidade de vida dos idosos, mas também de seus familiares e cuidadores. Referem-se aos transtornos da percepção, do conteúdo do pensamento, do humor e do comportamento (VEGA et al., 2007).

Segundo Tamai (2002), os sintomas comportamentais e psicológicos são divididos em dois agrupamentos psicopatológicos. O primeiro são os Sintomas
Comportamentais, que normalmente são identificados através da observação do paciente e incluem: agressão física, gritos, inquietação, agitação, perambulação, comportamentos culturalmente inapropriados e desinibição sexual. Já os Sintomas Psicológicos são avaliados, principalmente, com base nas entrevistas com os pacientes, seus familiares e cuidadores e incluem: ansiedade, humor deprimido, alucinações e delírios.

Esses sintomas (comportamentais e psicológicos) estão associados também, ao maior desgaste do cuidador, maior morbidade, ao declínio cognitivo mais rápido, elevação dos custos de tratamento e piora na qualidade de vida do paciente e de seu cuidador (FERRETTI, 2007; TAMAI, 2002).

Ainda que a institucionalização de idosos ocorra, principalmente, quando seus familiares/cuidadores, não conseguem mais assisti-los em suas necessidades, devido à dependência no desempenho de Atividades Básicas da Vida Diária (ABVD), os sintomas comportamentais e psicológicos, são alguns dos fatores responsáveis pela institucionalização precoce, devido ao estresse que causa tanto ao cuidador quanto ao idoso (CONVERSO; IARTELLI, 2007).

A dependência está relacionada diretamente com a necessidade de uma assistência. A literatura gerontológica consagrou a distinção entre cuidado formal e informal. O primeiro é oferecido por profissionais e o segundo por familiares, vizinhos e amigos. Porém, os cuidadores formais, que atuam, principalmente em instituições de longa permanência (ILP), nem sempre recebem treinamento e orientação sobre o que é cuidar (CARNEIRO et al., 2009).

O cuidar, segundo Carneiro et al. (2009), é uma ciência e como tal é necessária a preparação teórica e prática, capacitando os cuidadores formais, pois a falta de estudos interfere direta e indiretamente na prestação de cuidados aos idosos.

Sendo assim, a relação existente entre idosos com doenças crônicas não transmissíveis e crônico-degenerativas, sintomas comportamentais e psicológicos, institucionalização e necessidade de cuidadores formais são de fundamental relevância para a formulação de políticas públicas que favoreçam a melhoria na qualidade de vida de ambas as populações (idosos e cuidadores).

O presente estudo teve como objetivo identificar a freqüência e a intensidade dos sintomas comportamentais e psicológicos em idosos com diferentes doenças crônicas, residentes de uma Instituição de Longa Permanência, referidos por seus cuidadores formais, relacionando-os com o diagnóstico, a sobrecarga subjetiva e o desgaste proporcionado a esses cuidadores. 


\section{METODOLOGIA}

O projeto de pesquisa, incluindo o termo de consentimento livre e esclarecido (TCLE) obtiveram a aprovação do Núcleo de Bioética em Pesquisa da Universidade Federal de São Paulo - Campus Baixada Santista, através do número de protocolo 0160/10.

A coleta de dados ocorreu na Instituição de Longa Permanência (ILP) "Casa do Sol”, localizada em Santos, São Paulo. O levantamento de dados foi realizado entre os meses de abril e junho de 2010. Neste período, havia 98 moradores na instituição e 20 cuidadores formais, destes, 12 cuidadores aceitaram participar do estudo, respondendo por 90 idosos residentes.

Para o levantamento das idades e diagnósticos dos idosos foram acessados os bancos de dados administrativos e os prontuários clínicos.

Devido ao grande número de comorbidades presentes nos idosos, para fazer as análises estatísticas das mesmas, essas foram categorizadas, segundo o impacto sobre os sintomas, assim foram formadas 5 categorias de diagnóstico (de 1 a 5) tendo como base reconhecida influência dos mesmos nos sintomas comportamentais e psicológicos, ou seja, diagnósticos como HAS, DM, Cardiopatia, Osteoporose e Paralisia Infantil (PI) tiveram como valor de impacto nos sintomas 1; AVE, Síndrome de Down (SD) e Déficit de Memória, o valor atribuído foi 2; Depressão e Parkinson 3; Psicopatia e Transtorno Bipolar 4 e Demência e DA o valor foi 5.

Para a coleta de dados referentes à presença, intensidade e frequência dos sintomas comportamentais e psicológicos, nível de sobrecarga do cuidador e desempenho funcional dos idosos, foram aplicados, junto aos cuidadores, três instrumentos de avaliação: Inventário Neuropsiquiátrico
(INP) (CUMMINGS et al., 1994; CAMOZZATO et al., 2008); Inventário de Sobrecarga de Zarit (ISZ) (ZARIT et al., 1985; TAUB et al., 2004) e a Escala de Independência em Atividades da Vida Diária (Escala de Katz) (KATZ et al., 1970; LINO et al., 2008).

O INP (CUMMINGS et al., 1994; CAMOZZATO et al., 2008) consiste na avaliação de doze sintomas comportamentais e psicológicos. A freqüência dos sintomas é pontuada em uma escala de 1 (uma vez por semana) a 4 (uma vez por dia ou mais) pontos, a intensidade de 1 (leve) a 3 (acentuada) pontos e o desgaste do cuidador de 0 (nenhum) a 5 (muito acentuado ou extremo) pontos. A pontuação total pode variar de 0 a 144 pontos (excluindo-se a pontuação do desgaste, que é realizada separadamente) quanto maior a pontuação maior a frequência e a intensidade dos sintomas.

O ISZ (TAUB et al., 2004; ZARIT et al., 1985) é um instrumento utilizado para medir a sobrecarga subjetiva do cuidador, composto por 22 questões, pontuadas em uma escala de 0 (nunca) a 4 (quase sempre), de acordo com a presença ou intensidade da sobrecarga (TAUB et al., 2004).

Um escore total é calculado e a sobrecarga subjetiva é considerada severa para escores de 61 a 88, moderada para 41 a 60, leve para 21 a 40 e mínima ou ausente para escores abaixo de 20 (TAUB et al., 2004).

Para esse estudo, este inventário foi adaptado, visto que é um instrumento direcionado a cuidadores familiares e os sujeitos que participaram deste estudo são cuidadores formais.

Do total de 22 perguntas, duas foram excluídas por se tratarem de questões de cunho familiar e cinco foram modificadas para adaptação ao contexto do cuidador formal e nas 15 questões restantes a palavra familiar foi substituída por idoso. O Quadro 1 apresenta as modificações realizadas.

Quadro 1. Modificações realizadas no ISZ para aplicação com os cuidadores formais da instituição

\begin{tabular}{|l|l|}
\hline Instrumento original & Modificações/Adaptações \\
\hline $\begin{array}{l}\text { Você sente que não tem tanta privacidade como gostaria por } \\
\text { causa do seu familiar? } \\
\begin{array}{l}\text { Você se sente desconfortável em receber amigos em casa } \\
\text { devido ao seu familiar? }\end{array}\end{array}$ & Ambas as questões foram excluídas do instrumento. \\
\hline $\begin{array}{l}\text { Você sente que não tem tempo suficiente para você devido } \\
\text { ao tempo que dedica ao seu familiar? }\end{array}$ & $\begin{array}{l}\text { Você sente que não tem tempo suficiente para realizar outras tarefas de } \\
\text { sua função na instituição devido ao tempo que dedica ao idoso? }\end{array}$ \\
\hline $\begin{array}{l}\text { Você sente que seu familiar frequentemente afeta negativa- } \\
\text { mente seu relacionamento com outros membros da família? }\end{array}$ & $\begin{array}{l}\text { Você sente que o idoso frequentemente afeta negativamente o seu } \\
\text { relacionamento com os outros membros da equipe? }\end{array}$ \\
\hline $\begin{array}{l}\text { Você sente que sua vida social foi prejudicada por causa } \\
\text { dos cuidados com o seu familiar? }\end{array}$ & $\begin{array}{l}\text { Você sente que o idoso parece esperar que você cuide dele(a) como se } \\
\text { você fosse o(a) único(a) de quem ele(a) pudesse depender? }\end{array}$ \\
\hline $\begin{array}{l}\text { Você sente que não tem dinheiro suficiente para cuidar de } \\
\text { seu familiar, além de outras despesas? }\end{array}$ & $\begin{array}{l}\text { Você sente que o seu salário não equivale a sua dedicação neste traba- } \\
\text { lho? }\end{array}$ \\
\hline $\begin{array}{l}\text { Você sente que perdeu o controle de sua vida desde que seu } \\
\text { familiar adoeceu? }\end{array}$ & $\begin{array}{l}\text { Você sente que deixou de realizar muitas atividades de lazer desde que } \\
\text { iniciou neste trabalho? }\end{array}$ \\
\hline
\end{tabular}


Foi realizado o pré-teste, da versão adaptada, com seis funcionários da instituição, visando identificar a compreensão e a interpretação das questões, possibilitando a identificação de erros e dificuldades de compreensão e se o questionário adaptado mapeava as informações de interesse para o estudo. Os funcionários não relataram qualquer dificuldade, dizendo ser um questionário de fácil aplicação e entendimento.

Segundo Guerra (2000) o desgaste é compreendido como alterações orgânicas e psíquicas que um sujeito pode apresentar devido a fatores internos como alterações fisiológicas decorridas da idade e fatores externos. Já a sobrecarga subjetiva para Platt (1985 apud CAMPOS; SOARES, 2005) refere-se aos sentimentos pessoais sobre o ato de cuidar. Devido a diferenciação entre os conceitos justifica-se a necessidade de escalas diferentes para a avaliação de ambos (ISZ para sobrecarga subjetiva e INP para desgaste).

A Escala de Katz (KATZ et al., 1970; LINO et al., 2008) é um instrumento composto por seis itens que avaliam o desempenho do indivíduo em ABVD, como: tomar banho, vestir-se, uso do vaso sanitário, transferência, continência e alimentação. Cada item contem alternativas classificadas como independente ou dependente. A pontuação varia de 0 (independente em todas as funções) a 6 (dependente em todas as funções) pontos (LINO et al., 2008).

Os dados foram analisados por meio do programa estatístico SPSS (Statistical Package for the Social Sciences) versão 18.0.

Foi conduzida análise descritiva das informações demográficas (idade e gênero) e referentes a aplicação dos instrumentos (INP, Escala de Katz e ISZ), a partir do cálculo de medidas de tendência central e dispersão e cálculo das porcentagens, de acordo com tipo de variável (DORIA FILHO, 1999).

A partir da análise dos dados foram feitas correlações entre as pontuações totais dos instrumentos de avaliação (coeficiente de correlação de Pearson), assim como entre as pontuações totais e as variáveis dos INP e da Escala de Katz (coeficiente de correlação de Spearman), para identificar quais as variáveis que interferem na pontuação total dos instrumentos. O grau de significância foi fixado para valores de $p<0,05$ (DORIA FILHO, 1999).

\section{RESULTADOS}

A Tabela 1 apresenta o perfil demográfico dos idosos moradores da instituição e dos cuidadores formais que participaram do estudo, especificamente para gênero e idade.

Tabela 1. Análise descritiva e demográfica dos idosos moradores da instituição e dos cuidadores formais que participaram do estudo

\begin{tabular}{l|l|l|l|l|l|l|l}
\hline & N & Idade & Média & Desvio Padrão & Gênero & Feminino & \multirow{2}{*}{ Masculino } \\
\hline \multirow{2}{*}{$\begin{array}{l}\text { Idosos } \\
\text { Cuidadores }\end{array}$} & 90 & & 79,80 & 9,40 & & $71,1 \%$ & $28,9 \%$ \\
\cline { 2 - 6 } & 12 & & 30,42 & 5,31 & & $75 \%$ & $25 \%$ \\
\hline
\end{tabular}

Verificou-se alta prevalência do gênero feminino entre os idosos moradores e os cuidadores formais.

A Tabela 2 apresenta os diagnósticos mais comuns entre os idosos moradores da instituição, que participaram do estudo.

Todos os idosos participantes deste estudo apresentaram no mínimo um diagnóstico de doença crônica, sendo a HAS a mais prevalente. Vale ressaltar, que o total excede $100 \%$, uma vez que, a maioria dos idosos apresentavam mais de um diagnóstico de doença crônica.

Identificou-se que $37,8 \%$ dos idosos avaliados neste estudo foram considerados independentes. A dependência parcial, determinada por pontuações de 1 a 5 , correspondeu a 56,6\% dos idosos e a dependência total (pontuação 6), a $5,6 \%$ dos idosos.
Tabela 2. Diagnósticos dos idosos moradores da instituição

\begin{tabular}{l|c}
\hline \multicolumn{1}{c|}{ Diagnóstico } & \% \\
\hline Hipertensão Arterial Sistêmica (HAS) & 46,6 \\
Diabetes Melittus (DM) & 35,5 \\
Demência & 26,6 \\
Doença de Alzheimer (DA) & 21,1 \\
Cardiopatia & 11,1 \\
Acidente Vascular Encefálico (AVE) & 8,9 \\
Depressão & 8,9 \\
Outros diagnósticos & 23,3 \\
\hline
\end{tabular}

As atividades de vestir-se (52,2\%) e tomar banho $(45,6 \%)$ foram as mais pontuadas com a categorização máxima para dependência (4), enquanto alimentação $(85,6 \%)$ e transferência $(65,6 \%)$ foram as mais pontuadas 
com o nível máximo de independência (1).

A Tabela 3 apresenta os sintomas comportamentais e psicológicos mais presentes, freqüentes, intensos e desgastantes para os cuidadores formais em relação aos idosos, segundo os resultados obtidos com a aplicação do INP.

Tabela 3. Sintomas comportamentais e psicológicos mais presentes, frequentes e intensos e desgaste dos cuidadores formais frente à aos comportamentos

\begin{tabular}{c|c|c}
\hline & Sintomas & $\%$ \\
\hline \multirow{3}{*}{ Mais Presentes } & Depressão & 52,2 \\
& Agitação & 43,3 \\
& Ansiedade & 42,2 \\
& Irritabilidade & 40 \\
& Delírios & 25,6 \\
\hline \multirow{3}{*}{ Mais Frequentes (ao menos uma vez ao dia) } & Ansiedade & 28,9 \\
& Agitação & 22,2 \\
& Depressão & 22,2 \\
& Delírios & 15,6 \\
& Irritabilidade & 14,4 \\
\hline \multirow{2}{*}{ Mais Intensos } & Ansiedade & 25,6 \\
& Agitação & 20 \\
& Depressão & 17,8 \\
& Irritabilidade & 14,4 \\
& Delírios & 13,3 \\
\hline \multirow{2}{*}{ Mais Desgastantes } & Irritabilidade & 7,8 \\
& Agitação & 6,7 \\
& Ansiedade & 5,6 \\
& Depressão & 4,4 \\
& Euforia & 3,3 \\
\hline
\end{tabular}

Em se tratando da pontuação total, a média para os idosos residentes da instituição foi 21,9 pontos no INP com mínima de 0 e máxima de 78 pontos.

A pontuação total do ISZ sugeriu que $66,7 \%$ dos cuidadores dos idosos residentes pontuaram entre 23 e 36, sendo considerado sobrecarga leve e $33,3 \%$, pontuaram entre 9 e 20, sendo considerado sobrecarga mínima ou ausente.

A Tabela 4 apresenta as correlações significativas encontradas entre os resultados totais da Escala de Katz, INP, o desgaste do cuidador avaliado pelo INP e o diagnóstico.
Tabela 4. Correlações significativas entre os resultados totais da Escala de Katz, INP, INP desgaste e os diagnósticos

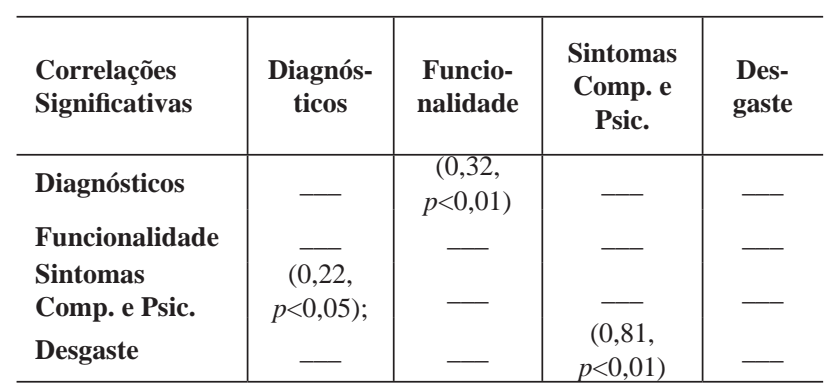


A Tabela 5 apresenta as correlações significativas encontradas entre algumas variáveis da Escala de Katz e do INP, com o resultado total do ISZ, INP e Escala de Katz.

Tabela 5 - Correlações significativas entre algumas variáveis da Escala de Katz e do INP com os resultados totais do ISZ, INP e Escala de Katz

\begin{tabular}{llll}
\hline $\begin{array}{l}\text { Correlações } \\
\text { Significativas }\end{array}$ & $\begin{array}{l}\text { Sobrecarga } \\
\text { Subjetiva }\end{array}$ & $\begin{array}{l}\text { Sintomas } \\
\text { Comp. e } \\
\text { Psic. }\end{array}$ & $\begin{array}{l}\text { Funciona- } \\
\text { lidade }\end{array}$ \\
\hline Banho & $(0,23, p<0,05)$ & - & - \\
Alimentação & $(0,21, p<0,05)$ & - & - \\
Continência & - & $(0,21, p<0,05)$ & - \\
Alucinações & - & & $(0,30$, \\
& & - & $p<0,01)$ \\
\hline
\end{tabular}

DISCUSSÃO

Os resultados encontrados neste estudo sobre o perfil demográfico dos cuidadores formais são semelhantes aos encontrados no estudo de Ribeiro et al. (2008) realizado com os cuidadores de idosos de ILP filantrópicas e privadas de Belo Horizonte. Demonstrando a predominância do gênero feminino e a média de idade inferior a 50 anos.

O cuidar muitas vezes é atribuído às mulheres, pois está diretamente relacionado com um dos seus principais papeis: o de ser mãe. A prática de cuidar dos filhos funciona como facilitadora na adaptação a esta função. Além disso, a relação afetiva das mulheres contribui para a humanização das instituições (CARNEIRO et al., 2008; RIBEIRO et al., 2008).

Nesta amostra de idosos ( $\mathrm{n}=90)$ foi possível identificar que o gênero feminino é predominante (71,1\%), tendência apontada por estudos relacionados com os idosos institucionalizados, em que a participação masculina é consideravelmente inferior à feminina (DAVIM et al., 2004; VIVAN; ARGIMON, 2009).

Isso ocorre devido à diferença com relação à expectativa de vida entre os gêneros. Atualmente, segundo a Síntese de Indicadores Sociais de 2009, espera-se que a mulher viva até os 77 anos, enquanto o homem até os 69,4 anos (IBGE, 2009; VIVAN; ARGIMON, 2009).

A média de idade entre os idosos foi de 79,8 anos, sendo que $64,4 \%$ dos moradores estão acima dos 80 anos. Estudo produzido por Mazza e Lefèvre (2004) demonstrou que a idade avançada é um dos principais fatores de institucionalização, isso porque o risco de adoecer e as chances do idoso tornar-se dependente são maiores.

Araújo e Ceolim (2007) citaram dois estudos (THORUN et al., 2001; MORAES et al., 2002 apud ARAÚJO; CEOLIM, 2007) em que a maior porcentagem dos idosos institucionalizados foram considerados independentes, através da Escala de Katz. No primeiro, $36,9 \%$ dos idosos foram considerados dependentes parciais e $13,1 \%$ dependentes totais, enquanto no segundo estudo $24 \%$ eram dependentes parciais e $29 \%$ dependentes totais.

Esta pesquisa apontou resultados diferentes, uma vez que a maior porcentagem foi atribuída à dependência parcial (56,6\%), seguida pela independência $(37,8 \%)$ e dependência total (5,6\%).

A capacidade funcional é um marcador de saúde da população idosa e pode ser compreendida como a potencialidade que o indivíduo tem de realizar as atividades da vida diária sem necessitar de ajuda (ALVES et al., 2007).

Rosa et al. (2003) demonstraram em seu estudo que as doenças crônicas estão associadas a incapacidade funcional do idoso, porém Alves et al. (2007) ressaltam que as diversas condições crônicas não tem o mesmo impacto na funcionalidade do idoso, podendo este ter mais de um diagnóstico e preservar a sua capacidade funcional, enquanto outro indivíduo com apenas um diagnóstico ter incapacidade funcional.

Existem recursos como a reabilitação que promovem a recuperação ou adaptação da capacidade comprometida, podendo o idoso retomar algumas atividades de seu repertório, incluindo as ABVD (ARAÚJO; CEOLIM, 2007).

Um aspecto relevante discutido por Araújo e Ceolim (2007) refere-se a situações em que os cuidadores formais estimulam a dependência dos idosos, como durante o banho e o vestuário, em que os moradores poderiam realizar a atividade, porém lentamente. "O fato é que mesmo o idoso independente, quando institucionalizado, pode desenvolver diferentes graus de dependência devido à dificuldade em aceitar e adaptar-se às novas condições de vida e à falta de motivação e de encorajamento que são comuns no ambiente asilar" (ARAÚJO; CEOLIM, 2007, p. 381).

Os mesmos autores relatam que a dependência comportamental é uma das mais temidas pelos idosos, e pode ocorrer devido ao desamparo em um ambiente não responsivo, negligenciador e não contingente (ARAÚJO; CEOLIM, 2007).

Em se tratando da pontuação total do INP, a média para os idosos residentes da instituição foi 21,9 pontos, com mínima de 0 e máxima de 78 . Não há na literatura nacional referência à nota de corte para este inventário, 
sabe-se que quanto maior a pontuação maior a freqüência e a intensidade dos sintomas, e o que se pode afirmar é que $86,7 \%$ dos idosos, referidos neste estudo, possuem ao menos um sintoma comportamental ou psicológico.

Observa-se que os estudos nacionais (CASSIS et al., 2007; FIALHO et al., 2009) apontam correlação significativa entre a presença dos SCPD, o comprometimento da funcionalidade e a sobrecarga subjetiva de seus respectivos cuidadores. Nos resultados encontrados neste estudo identificou-se que esses aspectos não se correlacionam, visto que a amostra era composta por idosos com diferentes diagnósticos de doenças crônicas e não apenas por idosos com demência como o estudo citado.

A pontuação total do INP apresentou correlação significativa com a pontuação total do desgaste, pontuada neste mesmo instrumento de avaliação $(\mathrm{r}=0,81, p<0,01)$. Essa correlação é considerada forte sugerindo que há relação entre os sintomas e o desgaste relatados pelos cuidadores formais da instituição.

Tais resultados sugerem a necessidade de instruir os cuidadores formais com relação a como lidar com a presença dos sintomas comportamentais e psicológicos, pois estes são responsáveis pelo desgaste proporcionado a esses profissionais, que pode ser evitado com o treinamento correto e com adequado apoio psicológico, uma vez que a sobrecarga subjetiva está diretamente relacionada às questões emocionais, favorecendo assim a melhora da qualidade do serviço prestado.

Obteve-se correlação significativa entre o diagnóstico e a pontuação total do INP $(\mathrm{r}=0,22, p<0,05)$, assim como entre a pontuação total da Escala de Katz e o diagnóstico ( $\mathrm{r}=0,32$, $p<0,01)$. Estes resultados sugerem que há relação entre o diagnóstico apresentado pelos idosos referidos no estudo e a presença dos sintomas comportamentais e psicológicos, assim como com o prejuízo da funcionalidade.

Alves et al. (2007) avaliaram a influência das doenças crônicas na capacidade funcional de idosos do município de São Paulo, concluindo que doenças pulmonares, artropatias, doenças cardíacas e HAS tem uma forte influência nas ABVD e AIVD. Os autores citaram ainda estudos (BARDAGE; ISACSON, 2001; WENSING et al., 2001; REKENEIRE et al., 2003 apud ALVES et al., 2007) que comprovam a relação da DM com o declínio funcional.

Em se tratando da sobrecarga subjetiva dos cuidadores formais e os domínios da Escala de Katz foram encontradas correlações significativas com a atividade de alimentação $(\mathrm{r}=0,21, \mathrm{p}<0,05)$ e a atividade de tomar banho $(0,23, p<0,05)$

Não há estudos nacionais que abordem a correlação existente entre os domínios da Escala de Katz e a sobrecarga do cuidador formal. Sugere-se uma hipótese para tais correlações: a atividade de tomar banho foi a segunda atividade em que os idosos foram considerados mais dependentes (a primeira foi vestir-se) e por isso maior a sobrecarga subjetiva, enquanto que a alimentação foi atividade em que os idosos foram considerados mais independentes, sugerindo que a minoria de idosos que são dependentes para essa atividade acarretam uma maior sobrecarga subjetiva para os cuidadores formais, visto que alimentar outra pessoa é uma atividade que acarreta um tempo elevado, assim como paciência.

O estímulo a independência e a autonomia do idoso, em ILP, torna-se cada vez mais raro e é a base para a manutenção da capacidade funcional e independência comportamental (ARAÚJO; CEOLIM, 2007).

Em se tratando de idosos que possuem doenças crônicas, que em sua maioria comprometem o desempenho funcional, é de fundamental importância o estímulo a independência e autonomia dos mesmos.

\section{CONCLUSÕES}

Pode-se afirmar que 86,7\% dos idosos residentes apresentavam ao menos um sintoma, os mais comuns nos idosos participantes do estudo foram: depressão (52,2\%), agitação (43,3\%) e ansiedade (42,2\%), os mais freqüentes foram: ansiedade (28,9\%), agitação e depressão (ambos com $22,2 \%$ ), assim como os mais intensos $25,6 \%, 20 \%$ e $17,8 \%$, respectivamente.

Irritabilidade (7,8\%), agitação (7,8\%) e ansiedade $(5,6 \%)$ foram os sintomas consideramos mais desgastantes aos cuidadores formais. Com relação a sobrecarga subjetiva dos cuidadores formais obteve-se que $66,7 \%$ apresentam sobrecarga leve e 33,3\% mínima ou ausente.

Com relação a funcionalidade observou-se que $37,8 \%$ dos idosos foram considerados independentes, $41,1 \%$ dependentes parciais e 5,6\% dependentes totais. Observouse maior dependência nas atividades de vestuário e banho e maior independência em alimentação e transferência.

Foram encontradas correlações significativas entre o diagnóstico e a funcionalidade e entre o diagnóstico e os sintomas comportamentais e psicológicos.

Outras correlações significativas encontradas foram: entre os sintomas e o desgaste do cuidador formal, entre a sobrecarga subjetiva e as atividades de banho e alimentação, entre os sintomas e atividade de continência e entre a funcionalidade e as alucinações.

Os resultados encontrados neste estudo podem estar relacionados com a forma como foram obtidas 
as informações, uma vez que os cuidadores formais que responderam levaram em consideração suas percepções, conhecimentos e informações.

Uma limitação do estudo se relaciona ao tamanho da amostra (apenas uma instituição) que não permite a generalização dos resultados.

Esta pesquisa demonstrou a importância das intervenções sobre os sintomas comportamentais e psicológicos, considerando sua repercussão para a capacidade funcional. Esta capacidade compõe o paradigma de saúde para a população idosa, e orienta a prestação do cuidado. Assim, os cuidadores informais devem ser instruídos sobre o que são os sintomas comportamentais e psicológicos, e sobre o desgaste e sobrecarga subjetiva que acarretam.

Agradecimentos: A Instituição de Longa Permanência Casa do Sol. As Terapeutas Ocupacionais Camilla Pádua Lima, Nathália Barros de Andrade e Ana Flávia Izumi pela contribuição com a coleta de dados desta pesquisa. Não há conflito de interesse entre os autores em relação ao presente artigo.

CANON, M. B. F.; NOVELLI, M. M. P. C. Identification of Behavioral and Psychological Symptoms in the residents elderly of a Long Stay Institution. Rev. Ter. Ocup. Univ. São Paulo, v. 23, n. 1, p. 72-80, jan./abr. 2012.

\begin{abstract}
Chronic diseases affect significantly the quality of life of elderly population and may also be associated with the presence of behavioral and psychological symptoms. The aim of this study was to identify the frequency and intensity of behavioral and psychological symptoms in institutionalized elderly, referred by their formal caregivers, relating them with the diagnosis, subjective burden and the wear proportionate to the formal caregiver. Three assessment tools were applied to formal caregivers of Nursing Homes: the Neuropsychiatric Inventory, The Independence in Activities of Daily Living Index (Katz Index) and the Zarit Caregiver Burden Interview. Twelve formal caregivers were evaluated, responding by 90 elderly residents of the institution, $86.7 \%$ of the elderly presented at least one symptom, the most common were: anxiety (28.9\%), agitation and depression (both with 22.2\%), as well as the most intense 25.6\% (anxiety), 20\% (agitation) and $17.8 \%$ (depression) tively. Significant correlations were found between the diagnosis and the functionality and between diagnosis and symptoms. This research demonstrated the importance of interventions on behavioral and psychological symptoms and the attention to the caregiver, with relation to the information and the wear and subjective burden that symptoms attribute. Also noteworthy that the presence of these symptoms contributes to the reduction of the functional capacity, which is a marker of health for the elderly population.
\end{abstract}

KEYWORDS: Aged; Homes for the aged; Chronic disease; Behavioral symptoms/psychology; Caregivers/psychology; Questionnaires/utilization.

\section{REFERÊNCIAS}

ALVES, L. C.; LEIMANN, B. C. Q.; VASCONCELOS, M. E. L.; et al. A influência das doenças crônicas na capacidade funcional dos idosos do Município de São Paulo, Brasil. Cad Saúde Pública, v.23, n. 8, p. 1924-1930, 2007.

AMERICAM PSYCHIATRIC ASSOCIATION (APA). Diagnostic and statistical manual of mental disorders. 4th. ed. revised. Washington (DC), 2000.

ARAÚJO, M. O. P. H.; CEOLIM, M. F. Avaliação do grau de independência de idosos residentes em instituições de longa permanência. Rev. Esc. Enferm. USP, v.41, n. 3, p. 378-385, 2007.

CAMOZZATO, A. L.; KOCHHANN, R.; SIMEONI, C.; et al. Reliability of the Brazilian Portuguese version of the Neuropsychiatric Inventory (NPI) for patients with Alzheimer's disease and their caregivers. Int. Psychogeriat., v. 20, p. 383-393, 2008.

CAMPOS, P. H. F.; SOARES, C. B. Representação da sobrecarga familiar e adesão aos serviços alternativos em saúde mental. Psicol. Rev., v. 11, p. 219-237, 2005.

CARNEIRO, B. G.; PIRES, E. O.; DUTRA FILHO, A. D.; GUIMARÃES, E. A. Perfil dos cuidadores de idosos de instituições de longa permanência de sintomatologia dolorosa. ConScientiae Saúde, v. 8, p. 75-82, 2009.

CASSIS, S. V. A.; KARNAKIS, T.; MORAES, T. A.; et al. 
Correlação entre o estresses do cuidador e as características clínicas do paciente portador de demência. Rev. Assoc. Med. Bras., v. 53, p. 497-501, 2007.

CONVERSO, M. E. R.; IARTELLI, I. Caracterização e análise do estado mental e funcional de idosos institucionalizados em instituições públicas de longa permanência. J. Bras. Psiquiatr., v. 56, p. 267-272, 2007.

CUMMINGS, J. L.; MEGA, M.; GARY, K. The neuropsychiatric inventory: comprehensive assessment of psychopathology in dementia. Neurology, v. 44, p. 2308-2314, 1994.

DAVIM, R.; TORRES, G.; DANTAS, S.; LIMA, V. Estudo com idosos de instituições asilares no município de Natal/RN: características socioeconômicas e de saúde. Rev. Latinoam. Enferm., v. 12, p. 518-524, 2004.

DORIA FILHO, U. Introdução a bioestatística: para simples mortais. São Paulo: Negócio Editora, 1999.

FERRETTI, R. E. L. Manejo não-farmacológico nas demências e intervenções ambientais. In: FORLENZA, O. Psiquiatria geriátrica: do diagnóstico precoce à reabilitação. São Paulo: Atheneu, 2007.

FIALHO, P. P. A.; KOENIG, A. M.; SANTOS, E. L.; et al. Dementia caregiver burden in Brazilian sample: Association to neuropshychiatric symptoms. Dementia Neuropsychol., v. 3, n. 2, p. 132-135, 2009.

GUERRA, C. I. C. O. Fatores de risco para desgaste do cuidador familiar de paciente na fila do transplante cardíaco. 2000. Dissertação (Mestrado). Escola de Enfermagem, Universidade de São Paulo, São Paulo, 2000.

INSTITUTO BRASILEIRO DE GEORGRAFIAE ESTATÍSTICA (IBGE). Síntese de indicadores sociais 2009. Rio de Janeiro, 2009. Disponível em: <http://www.ibge.gov.br>. Acesso em: 23 jul. 2010.

KATZ, S.; DOWNS, T. D.; CASH, H. R.; GROTZ, R. C. Progress in development of the index of ADL. Gerontologist, v. 10, p. 20-30, 1970

LINO, V. T. S.; PEREIRA, S. R. M.; CAMACHO, L. A. B.; et al. Adaptação transcultural da Escala de Independência em Atividades da Vida Diária (Escala de Katz). Cad. Saúde Pública, p. 103-112, 2008.

Recebido para publicação: 16/06/2011

Aceito para publicação: 20/12/2011
MARIN, M. J. S.; ANGERAMI, E. L. S. Caracterização de um grupo de idosas hospitalizadas e seus cuidadores visando o cuidado pós-alta hospitalar. Rev. Esc. Enferm. USP, v. 36, n. 1, p. 33-41, 2002.

MAZZA, M. M. P. R.; LEFÈVRE, F. A instituição asilar segundo o cuidador familiar do idoso. Saúde Sociedade, v. 13, p. 68-77, 2004.

NOGUEIRA, S. L.; GERALDO, J. M.; MACHADO, J. C.; RIBEIRO, R. C. L. Distribuição espacial e crescimento da população idosa nas capitais brasileiras de 1980 a 2006: um estudo ecológico. Rev. Bras. Estud. Popul., v. 25, p. 195-198, 2008.

ORGANIZAÇÃO MUNDIAL DA SAÚDE (OMS). Prevenção de doenças crônicas como um investimento vital. OMS, 2005.

PAIXÃO Jr, C. M.; REICHENHEIM, M. E. Uma revisão sobre instrumentos de avaliação do estado funcional do idoso. Cad. Saúde Publica, v. 21, p. 7-19, 2005.

RIBEIRO, M. T. F.; FERREIRA, R. C.; FERREIRA, E. F.; et al. Perfil dos cuidadores de idosos nas instituições de longa permanência de Belo Horizonte, MG. Cien. Saúde Coletiva, v. 13, n. 4, p.1285-1292, 2008.

TAMAI, S. Tratamento dos transtornos do comportamento de pacientes com demência. Rev. Bras. Psiquiatr., v. 24, p. 15-21, 2002.

TAUB, A.; ANDREOLI, S. B.; BERTOLUCCI, P. H. Dementia caregiver burden: reliability of the Brazilian version of the Zarit caregiver burden interview. Cad. Saude Publica, v. 20, p. 372376, 2004.

VEGA, U. M.; MARINHO, V.; ENGELHARDT, E.; LAKS, J. Sintomas neuropsiquiátricos nas demências: relato preliminar de uma avaliação prospectiva em um ambulatório do Brasil. Arq. Neuropsiquiatr., v. 65, p. 498-502, 2007.

VIVAN, A. S.; ARGIMON, I. I. L. Estratégias de enfrentamento, dificuldades funcionais e fatores associados em idosos institucionalizados. Cad. Saude Publica, v. 25, n. 2, p. 436-444, 2009.

ZARIT, S. H.; ORR, N. K.; ZARIT, J. M. The hidden victims of Alzheimer's disease; families under stress. New York: New York University Press, 1985. 